\title{
Comparing Syndromic Surveillance and Poison Center Data for Snake Bites in Missouri
}

\author{
Karen H. Pugh*1, Amy Kelsey ${ }^{2}$ and Rebecca Tominack ${ }^{3,1}$ \\ 1St. Louis University School of Medicine and School of Public Health, Saint Louis, MO, USA; ${ }^{2}$ Missouri Department of Health and \\ Senior Services, Jefferson City, MO, USA; ${ }^{3}$ Missouri Poison Center, Saint Louis, MO, USA
}

\section{Objective}

This study intends to use two different surveillance systems available in Missouri to explore snake bite frequency and geographic distribution.

\section{Introduction}

In 2010, there were 4,796 snake bite exposures reported to Poison Centers nationwide (1). Health care providers frequently request help from poison centers regarding snake envenomations due to the unpredictability and complexity of prognosis and treatment. The Missouri Poison Center (MoPC) maintains a surveillance database keeping track of every phone call received. ESSENCE, a syndromic surveillance system used in Missouri, enables surveillance by chief complaint of 84 different emergency departments (ED) in Missouri (accounting for approximately $90 \%$ of all ED visits statewide). Since calling a poison center is voluntary for health care providers, poison center data is most likely an underestimation of the true frequency of snake envenomations. Comparing MoPC and ESSENCE data for snake envenomations would enable the MoPC to have a more accurate depiction of snake bite frequency in Missouri and to see where future outreach of poison center awareness should be focused.

\section{Methods}

Archived data from Toxicall ${ }^{\circ}$, the MoPC surveillance system, was used to query the total number of snake bite cases from 01/01/2007 until 12/31/2011 called into the MoPC center by hospitals that also participate ESSENCE. Next, ESSENCE data was used to estimate the total number of snake envenomations presenting to EDs in Missouri. This was accomplished using the same date range as well as searching for key terms in the chief complaints that would signify a snake bite. The results of each datasearch were compared and contrasted by Missouri region.

\section{Results}

The Toxicall ${ }^{\circledR}$ search showed a total of 324 snake bite cases. The initial ESSENCE data query showed a total of 1983 snake bite cases. After certain data exclusions, there was a total of 1763 ESSENCE snake bite visits. This suggests that approximately $18 \%$ of all snake bite visits reported in Missouri ESSENCE were called into the MoPC. The results are demonstrated by Missouri region in Figure 1. This figure also shows that the greatest number of ESSENCE visits for snake bites were reported by Southwest region hospitals whereas the Eastern region hospitals placed the greatest number of calls to MoPC regarding snake bites.

\section{Conclusions}

The total number of snake bite cases from Missouri ESSENCE ED visits is much greater than the number of snake bites cases called into the MoPC by ESSENCE participating hospitals. This underutilization of the poison center demonstrates the increased need for awareness of the MoPC's free services. In Missouri, the MoPC should target hospitals in the Southwest region for outreach in particular based on these findings. Poison centers are staffed by individuals trained in all types of poisonings and maintain a list of consulting physicians throughout the United States experienced in management and treatment of venomous snake bites (2). Any heathcare facility would benefit from MoPC assistance. Finally, syndromic surveillance allows for quick and easy data compilation, however there are some difficulties when attempting to search for a particular condition. Communication and partnership between two different public health organizations will be beneficial toward future public health studies.

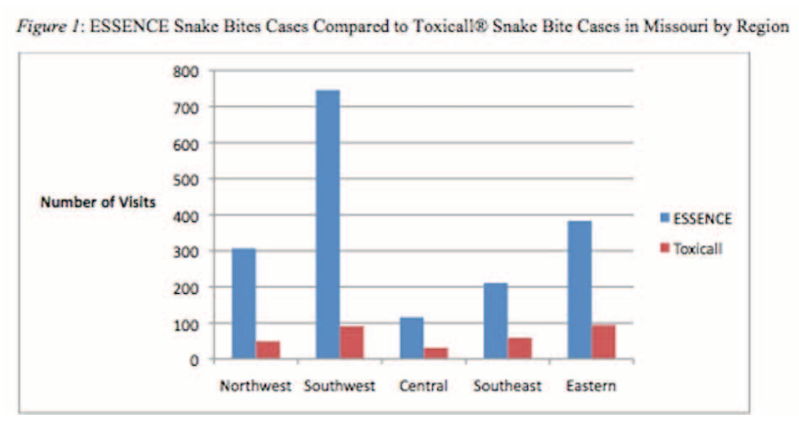

\section{Keywords}

ESSENCE; surveillance; Missouri; Poison Center; snake

\section{References}

1. Bronstein AC, Spyker DA, Cantilena LR, Green JL, Rumack BH, Dart RC. 2010 Annual report of the American Association of Poison Control Centers' national poison data system (NPDS): 28th annual report. Clinical Toxicology. 2011;49:910-41.

2. Gold BS, Barish RA, Dart RC. North American snake envenomation: diagnosis, treatment, and management. Emerg Med Clin N Am. 2004;22:423-43

\section{*Karen H. Pugh}

E-mail: khill9@slu.edu 